\title{
Variability of winds in X-ray binaries
}

\author{
M. Díaz Trigo ${ }^{1}$, L. Boirin ${ }^{2}$, S. Migliari ${ }^{3}$, J. Miller-Jones ${ }^{4}$, A. Parmar ${ }^{5}$ \\ and L. Sidoli ${ }^{6}$ \\ ${ }^{1}$ ESO, Karl-Schwarzschild-Strasse 2, D-85748 Garching bei München, Germany \\ email: mdiaztri@eso.org \\ ${ }^{2}$ Observatoire Astronomique de Strasbourg, 11 rue de l'Université, F-67000 Strasbourg, France \\ ${ }^{3}$ DAM and ICC (IEEC-UB), Universitat de Barcelona, Mart i Franqus 1, \\ 08028 Barcelona, Spain \\ ${ }^{4}$ International Centre for Radio Astronomy Research, Curtin University, GPO Box U1987, \\ Perth, WA 6845, Australia \\ ${ }^{5}$ ESAC, P.O. Box 78, E-28691 Villanueva de la Cañada, Madrid, Spain \\ ${ }^{6}$ INAF-IASF, via Bassini 15, I-20133 Milano, Italy
}

\begin{abstract}
We discuss the variability of winds in two low-mass X-ray binaries, GX $13+1$ and $4 \mathrm{U} 1630-47$. XMM-Newton observations of these sources show that strong, photoionised winds with column densities well above $10^{22} \mathrm{~cm}^{-2}$ can be present in both neutron star and black hole LMXBs. Such winds can fade significantly due to changes in the flux or spectral hardness of the continuum. In particular, a decrease of column density and/or an increase of ionisation are measured when the flux increases or the spectrum hardens. We show how variability studies can help us to understand what triggers changes in the wind and discuss the limitations of current instruments for such studies.
\end{abstract}

Keywords. X-rays: binaries accretion, accretion disks - Stars: neutron - Black hole physics Spectroscopy.

\section{Introduction}

The presence of highly ionised plasma in a cylindrical geometry around the compact object has been observed in all the high-inclination neutron star (NS) and black hole (BH) low mass X-ray binaries (LMXBs) (Díaz Trigo et al. 2006, Ponti et al. 2012) i.e. when looking close to the disc. Narrow absorption features, identified with resonant absorption from Fe XXV and Fe XXVI and other abundant ions, are the most common signature of the highly ionised plasma. These features were first detected with ASCA from the microquasars GROJ1655-40 (Ueda et al. 1998; Yamaoka et al. 2001) and GRS 1915+105 (Kotani et al. 2000; Lee et al. 2002). Medium to high resolution spectra from Chandra, XMM-Newton and Suzaku showed that such absorption features are common to NS and $\mathrm{BH}$ binaries and probably associated to the accreting nature of those systems (e.g. Jimenez-Garate et al. 2002, Boirin et al. 2003, 2004, 2005, Díaz Trigo et al. 2006, 2007, King et al. 2012, Kubota et al. 2007, Miller et al. 2004, 2006, 2011, Sidoli et al. 2001, Ueda et al. 2004, 2009, Schulz et al. 2008). Therefore, studies of photoionised plasmas allow us to learn about accretion processes in LMXBs by e.g. mapping changes in the disc via changes in the disc atmosphere (or photoionised plasma above the disc).

The photoionised plasma has been detected as an outflowing wind in $85 \%$ of $\mathrm{BH}$ and in $30 \%$ of NS systems and as a static atmosphere in the rest of the cases (Díaz Trigo \& Boirin 2012). Therefore the amount of mass expelled could be very different for NSs and $\mathrm{BHs}$, or even for different accretion rates. This has important consequences for the impact of winds on the LMXBs themselves (e.g. in the disc structure) and on their 
environment. In particular, for systems showing winds the whole energy budget could be significantly altered by the mass expelled in the wind and have an effect on the dynamics of the accretion flow. In addition, feedback to the environment could be relevant if the outflows carry a significant amount of kinetic energy and momentum. Hence it is crucial to determine under which conditions a wind is launched and the magnitude of the wind mass outflows. Studies of the wind variability at different accretion rates and states and for different types of compact objects can hold the answer to these questions. In this work we present the results of such studies for two LMXBs.

\section{Results}

We focus here on recent XMM-Newton observations of two LMXBs, a NS and a BH, known to show strong winds (Ueda et al. 2004, Kubota et al. 2007). As a diagnostic tool for this study we concentrate on the narrow absorption lines and study their variability in several epochs.

GX $13+1$. We observed the persistent NS LMXB GX $13+1$ with XMM-Newton in five different epochs in 2008 to investigate the variability of the wind in this source (see Díaz Trigo et al. 2012 for details). As an example, Fig. 1 shows plots of the residuals from the best-fit continuum model around the Fe K region for two of the observations. Absorption features from highly ionised species of iron, such as Fe XXV and Fe XXVI K $\alpha$ and $\mathrm{K} \beta$ are evident. A broad iron emission line is present in all the observations. Both the absorption and emission features show significant variations among observations.

Taking into account the five epochs, we found a significant correlation between the hard, 6-10 keV, flux, the ionisation and column density of the absorber and the equivalent width of the broad iron line.

We interpreted the correlation in a scenario in which a disc wind is thermally driven at large, $\sim 10^{10} \mathrm{~cm}$, radii and the broad line results from reprocessed emission in the wind and/or hot atmosphere. The breadth of the emission line is naturally explained by a combination of scattering, recombination and fluorescence processes. We attribute the variations in the absorption and emission along the orbital period to the view of different parts of the wind, possibly located at slightly different inclination angles.

$4 U$ 1630-4\%. We observed the transient BH LMXB 4U 1630-47 with XMM-Newton in six different epochs during its soft-to-hard transition, at the end of its 2011-2012 outburst. As an example, Fig. 2 shows the residuals from the best-fit continuum model around the Fe $\mathrm{K}$ region for two different epochs.

Taking all the epochs into account, we observe a progressive drop of the column density of the wind with increasing hard X-ray flux. While strong, saturated, absorption lines of Fe XXV and Fe XXVI are visible in the first observation, the Fe XXV line has disappeared in the last observation and the Fe Xxvi line is barely detected.

\section{Discussion and outlook}

A comparison of the wind in GX $13+1$ and 4U 1630-47 shows interesting similarities. Both sources have winds with large column densities, $\gtrsim 10^{23} \mathrm{~cm}^{-2}$, which are revealed by saturated absorption lines and strong absorption edges (see Fig. 1 and 2, left panels). However, in both systems the column density of the wind can significantly drop together with an increase of ionisation or not (see Fig. 1 and 2, right panels). The changes in the wind could be triggered by changes in the flux or the spectral energy distribution. 

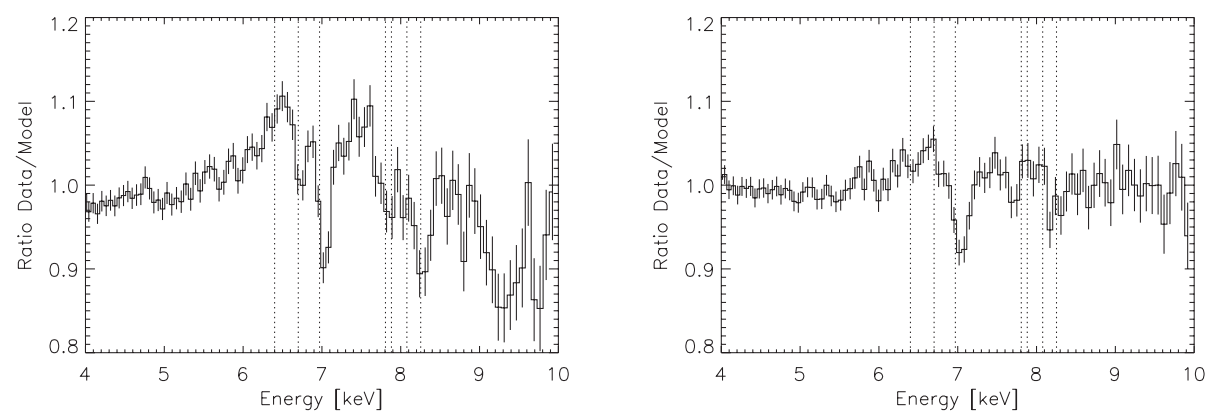

Figure 1. Ratio of the data to the continuum model at the Fe $\mathrm{K}$ region for two epochs of the XMM-Newton observations of GX 13+1. The dotted vertical lines indicate from left to right the rest energy of the transitions of neutral Fe, Fe xxv K $\alpha$, Fe xxvi K $\alpha$, Ni xxviI, Fe xxv K $\beta$, Ni xxviII and Fe XXvi K $\beta$.
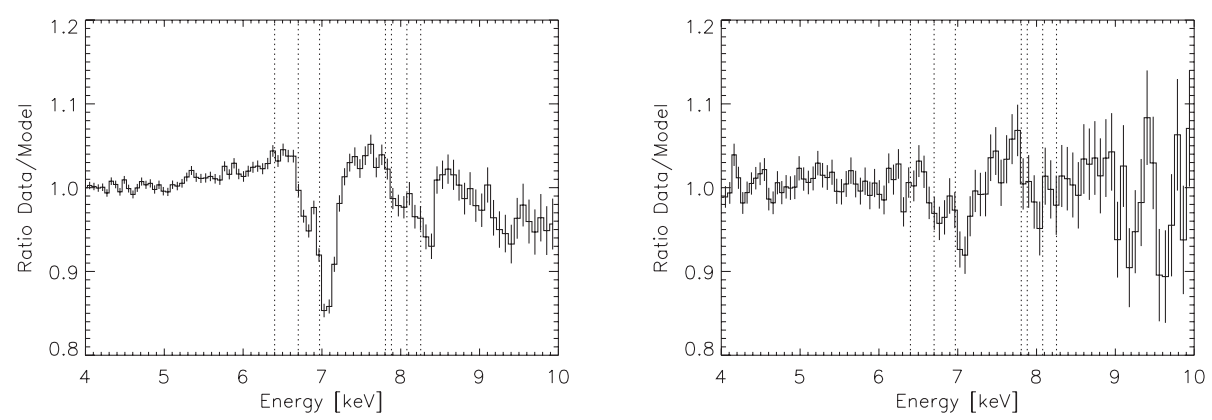

Figure 2. Ratio of the data to the continuum model at the Fe $\mathrm{K}$ region for two epochs of the XMM-Newton observations of $4 \mathrm{U} 1630-47$. The dotted vertical lines indicate from left to right the rest energy of the transitions of neutral Fe, Fe xxv K $\alpha$, Fe xxvi K $\alpha$, Ni xxvII, Fe Xxv K $\beta$, Ni XXVIII and Fe XXVI K $\beta$.

Ongoing studies of winds are aimed to understand to which extent a change of flux or spectral hardness can be responsible for the disappearance of a wind and under which circumstances a change in the accretion flow is needed (e.g. Ueda et al. 2009, Neilsen \& Homan 2012, Ponti et al. 2012). Sensitive simultaneous observations in X-ray and radio wavelengths of LMXBs throughout their outbursts together with an estimation of the expected winds at each state could soon shed some light on this topic.

It should be borne in mind that the best results for wind studies are obtained when several of the existent instruments are combined. For example, CCD cameras such as XMM-Newton/EPIC or Suzaku/XIS, capable of medium resolution spectroscopy, do not allow accurate measurements of the velocity shift of the lines, which is a fundamental parameter for determining the location of the wind and its launching mechanism. Instead, velocity shifts are best measured with high energy resolution gratings such as Chandra/HETGS or XMM-Newton/RGS.

In contrast, the column density of the wind is best determined with the EPIC-pn camera onboard XMM-Newton. The reason is that the large effective area above $7 \mathrm{keV}$ allows the simultaneous measurement of the $\mathrm{K} \alpha$ and $\mathrm{K} \beta$ components of $\mathrm{H}$ and He-like ions of $\mathrm{Fe}$ and of their absorption edges and this allows to determine the saturation of the lines when large column densities are present.

In addition, only Suzaku, with its broadband coverage from 0.3 to $600 \mathrm{keV}$, has the capability to measure the spectral energy distribution and therefore of disentangling its 
influence from the effect of an increase of bolometric luminosity for the appearance or disappearance of winds.

Future observations of winds with high resolution calorimeters, as the one onboard AstroH, will allow to determine with unprecedented accuracy the sites of wind production, thus further constraining the dynamics of accretion flows. In particular, it is expected that a calorimeter detector will resolve several components of the wind, which can only be done now in very deep high-resolution observations (e.g. Xiang et al. 2009, Kallman et al. 2009).

\section{References}

Boirin, L. \& Parmar, A. N., 2003, A\&A A, 407, 1079

Boirin, L., Parmar, A. N., Barret, D., Paltani, S., \& Grindlay, J. E. 2004, A\&GA, 418, 1061

Boirin, L., Méndez, M., Díaz Trigo, M., et al. 2005, A\&̈A, 436, 195

Díaz Trigo, M., Parmar, A. N., Boirin, L., et al. 2006, A\&A, 445, 179

Díaz Trigo, M., Parmar, A. N., Miller, J., et al. 2007, A\&A, 462, 657

Díaz Trigo, M., Sidoli, L., Boirin, L., \& Parmar, A. 2012, A\&A, 543, A50

Díaz Trigo, M. \& Boirin, L. 2012, Acta Polytechnica, in press, arXiv:1210.0318

Jimenez-Garate, M. A., Raymond, J. C., \& Liedhal, C. A. 2002, ApJ, 581, 1297

Kallman, T., Bautista, M., \& Goriely, S. 2009, ApJ, 865, 701

King, A., Miller, J. et al. 2012, ApJL, 746, L20

Kotani, T., Ebisawa, K., et al. 2000, ApJ, 539, 413

Kubota, A., Dotani, T., Cottam, J., et al. 2007, PASF, 59S, 185

Lee, J., Reynolds, C., et al. 2002, ApJ, 567, 1102

Miller, J., Raymond, J., et al. 2004, ApJ, 601, 450

Miller, J., Raymond, J., et al. 2006, Nature, 441, 953

Miller, J., Maitra, D., et al. 2011, ApJ, 731, L7

Neilsen, J. \& Homan, J. 2012, ApJ, 750, 27

Ponti, G., Fender, R. P., et al. 2012, MNRAS, 422, 11

Schulz, N. S., Kallman, T., et al. 2008, ApJ, 672, 1091

Sidoli, L., Oosterbroek, T., et al. 2001, A\&A, 379, 540

Ueda, Y., Inoue, H., et al. 1998, ApJ, 492, 782

Ueda, Y., Murakami, H., Yamaoka, K., et al. 2004, ApJ, 609, 325

Ueda, Y., Yamaoka, K., \& Remillard, R. 2009, ApJ, 695, 888

Xiang, J., Lee, J. C., et al. 2009, ApJ, 701, 984

Yamaoka, K., Ueda, Y., et al. 2001, PASJ, 53, 179 